\title{
OPPORTUNITIES IN THE UNITED STATES
}

T he US Epidemic Intelligence Service (EIS) annual conference and trainee assignment process could provide important models for the NSW Public Health Training Scheme.

The April conference, at the Centers for Disease Control (CDC) in Atlanta, Georgia, attracted a wide range of US public health professionals, including epidemiologists from most States and from the CDC.

My mission at the conference was to find out about EIS and then select a two-year training position, which begins this month. I came away with the strong belief that a local scientific meeting with emphasis on epidemiological investigations conducted by Public Health Trainees, the Epidemiology Branch and Public Health Units would provide a focus for building public health infrastructure in NSW.

The Communicable Disease Center was set up in 1946 by the United States Government, initially to investigate the malaria risk to people in the southern US. Over the next few years, that organisation developed into the Centers for Disease Control, which has since gained worldwide distinction for its epidemiological approach to public health problems. The CDC is now six centres, including:

\section{- Center for Prevention Services \\ - Center for Environmental Health and Injury Control \\ - National Institute for Occupational Health and Safety \\ 뭄 Center for Chronic Disease Prevention and Health Promotion \\ 밈 Center for Infectious Diseases \\ - National Center for Health Statistics}

CDC is perhaps best known in Australia for its weekly publication $M M W R$, which provides latest infectious diseases and mortality surveillance data to US health professionals. Through EIS, CDC offers both domestic and overseas health departments assistance in disease outbreak investigations. Each year CDC recruits about 75 health professionals to train in epidemiology, disease surveillance, investigation and prevention with EIS.
The EIS conference is an annual scientific meeting at which EIS trainees and alumni present reports of recent public health investigations. The papers at this year's conference were outstanding, with reports on investigations into areas including:

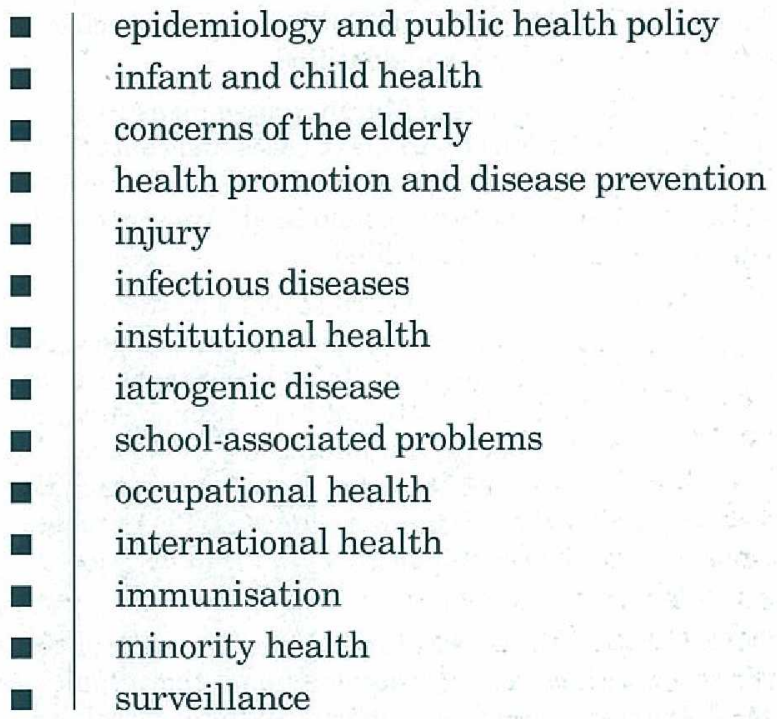

The quality of the papers was complemented by the high level of discussion that followed each presentation, probing epidemiological methodology and public health significance.

The conference also provided an opportunity for the 76 EIS recruits to meet epidemiologists offering training positions within the CDC or State health departments.

The formal EIS selection process was held the weekend after the conference. Each day recruits had around 2015 -minute timeslots in which to interview up to 150 epidemiologists offering training positions. After the interviews, recruits and supervisors ranked each other and, with the aid of a computer, the CDC assigned recruits to positions (mine is with the Oregon State Health Department).

My major impressions of CDC were the warmth of the staff and their commitment to public health and epidemiology.

Jeremy McAnulty

Public Health Officer 